\title{
LARGE DEVIATION PRINCIPLES OF ONE-DIMENSIONAL MAPS FOR HÖLDER CONTINUOUS POTENTIALS
}

\author{
HUAIBIN LI
}

\begin{abstract}
We show some level-2 large deviation principles for real and complex one-dimensional maps satisfying a weak form of hyperbolicity. More precisely, we prove a large deviation principle for the distribution of iterated preimages, periodic points, and Birkhoff averages.
\end{abstract}

\section{INTRODUCTION}

We consider large deviations for dynamical systems. It is well known that for uniformly hyperbolic dynamical systems the large deviation principle holds, and the rate functions are characterized by the differences between the metric entropies and the sums of positive Lyapunov exponents of invariant probability measures, see for example [Kif90, You90, and references therein. For nonuniformly hyperbolic dynamical systems there are some known results, see for example [AP06, CT12, Com09, CRL11, CTY13, KN92, Mel09, MN08, PS96, PS09a, PS09b, PSY98, RBY08, XF07, and references therein. See also the survey paper of Denker Den96.

This paper is devoted to the study of level-2 large deviation principles for Hölder continuous potentials of real or complex one-dimensional maps, under weak hyperbolicity assumptions. The first key observation is a result obtained by the author and Rivera-Letelier in [LRL12a that for a real or complex one-dimensional map satisfying such weak hyperbolicity assumptions, every Höder continuous potential has a unique equilibrium state, see Lemma 2.6. This, together with various formulas to compute the pressure function, allows us to apply [CRL11, Theorem C], which is a variant of a general result of Kifer in Kif90, Theorem 3.4], to obtain a full level-2 large deviation principles for sequences of measures associated to periodic points, iterated preimages, and Birkhoff averages.

We now proceed to describe our results in more detail.

1.1. Equilibrium states and level-2 large deviation priciple. Let ( $X$, dist) be a compact metric space and $T: X \rightarrow X$ a continuous map. Denote by $\mathcal{M}(X)$ the space of Borel probability measures on $X$ endowed with the weak* topology, and by $\mathcal{M}(X, T)$ the subspace of $\mathcal{M}(X)$ of those measures that are invariant by $T$. For each measure $\nu$ in $\mathcal{M}(X, T)$, denote by $h_{\nu}(T)$ the measure-theoretic entropy of $\nu$. For a continuous function $\varphi: X \rightarrow \mathbb{R}$, denote by $P(T, \varphi)$ the topological

2010 Mathematics Subject Classification. 60F10, 37E05, 37F10, 37D35.

Key words and phrases. Interval maps, rational maps, large deviation principles, Hölder continuous potentials .

The author was partially supported by the National Natural Science Foundation of China (Grant No. 11101124) and the FONDECYT grant 3110060 of Chile. 
pressure of $T$ for the potential $\varphi$, defined by

$$
P(T, \varphi):=\sup \left\{h_{\nu}(T)+\int_{X} \varphi d \nu: \nu \in \mathcal{M}(X, T)\right\} .
$$

A measure $\nu$ in $\mathcal{M}(X, T)$ is called an equilibrium state of $T$ for the potential $\varphi$, if the supremum in (1.1) is attained at $\nu$.

Recall that a sequence Borel probability measures $\left(\Omega_{n}\right)_{n \geq 1}$ on $\mathcal{M}(X)$ is said to satisfy a large deviation principle if there exists a lower semi-continuous function called a rate function $I: \mathcal{M}(X) \rightarrow[0,+\infty]$, such that for every closed subset $\mathcal{F}$ of $\mathcal{M}(X)$ we have

$$
\limsup _{n \rightarrow+\infty} \frac{1}{n} \log \Omega_{n}(\mathcal{F}) \leq-\inf _{\mathcal{F}} I
$$

and such that for every open subset $\mathcal{G}$ of $\mathcal{M}(X)$ we have,

$$
\liminf _{n \rightarrow+\infty} \frac{1}{n} \log \Omega_{n}(\mathcal{G}) \geq-\inf _{\mathcal{G}} I .
$$

Definition 1.1. Let ( $X$, dist) be a compact metric space and $T: X \rightarrow X$ be a continuous map. Let $\varphi: X \rightarrow \mathbb{R}$ be a Hölder continuous function. Moreover, for each integer $n \geq 1$ let $F_{n}: X \rightarrow \mathcal{M}(X)$ be the function defined by

$$
F_{n}(x):=\frac{1}{n} \sum_{i=0}^{n-1} \delta_{T^{i}(x)}=\frac{1}{n}\left(\delta_{x}+\delta_{T(x)}+\cdots+\delta_{T^{n-1}(x)}\right),
$$

and let $S_{n}(\varphi): X \rightarrow \mathbb{R}$ be defined by

$$
S_{n}(\varphi)(x):=\sum_{i=0}^{n-1} \varphi \circ T^{i}(x)=\varphi(x)+\varphi(T(x)) \cdots+\varphi\left(T^{n-1}(x)\right) .
$$

- Iterated preimages: Given $x_{0} \in X$, for each integer $n \geq 1$ put

$$
\Theta_{n}\left(x_{0}\right):=\sum_{y \in T^{-n}\left(x_{0}\right)} \frac{\exp \left(S_{n}(\varphi)(y)\right)}{\sum_{y^{\prime} \in T^{-n}\left(x_{0}\right)} \exp \left(S_{n}(\varphi)\left(y^{\prime}\right)\right)} \delta_{F_{n}(y)} .
$$

- Periodic points: Letting $P_{n}:=\left\{p \in X: T^{n}(p)=p\right\}$, put

$$
\Theta_{n}:=\sum_{p \in P_{n}} \frac{\exp \left(S_{n}(\varphi)(p)\right)}{\sum_{p^{\prime} \in P_{n}} \exp \left(S_{n}(\varphi)\left(p^{\prime}\right)\right)} \delta_{F_{n}(p)} .
$$

- Birkhoff averages: Assume there is a unique equilibrium state $\nu_{\varphi}$ of $T$ for the potential $\varphi$, put $\Sigma_{n}:=F_{n}\left[\nu_{\varphi}\right]\left(\right.$ i.e. the image measure of $\nu_{\varphi}$ by $\left.F_{n}\right)$.

We say the level-2 large deviation principle holds for $(T, \varphi)$ with respect to iterated preimages (resp. periodic orbits, Birkhoff averages) if there exist a unique equilibrium state $\nu_{\varphi}$ of $T$ for the potential $\varphi$, and if the sequences $\left(\Theta_{n}\left(x_{0}\right)\right)_{n \geq 1}$ (resp. $\left.\left(\Theta_{n}\right)_{n \geq 1},\left(\Sigma_{n}\right)_{n \geq 1}\right)$ converges to $\delta_{\nu_{\varphi}}$ in the weak* topology and satisfies a large deviation principle.

1.2. Statements of results. We state 2 results, one in the real setting and the other in the complex setting. To simplify the exposition, they are formulated in a more restricted situation than what we are able to handle, see the Main Theorem in 92 for a more general formulation of our results.

Given a compact interval $I$, a smooth map $f: I \rightarrow I$ is non-degenerate, if the number of points at which the derivative of $f$ vanishes is finite, and if for every such point there is a higher order derivative of $f$ that is non-zero. 
Theorem A. Let $I$ be a compact interval and let $f: I \rightarrow I$ be a topologically exact non-degenerate smooth map. Assume $f$ has only hyperbolic repelling periodic points, and for each critical value $v$ of $f$ we have

$$
\lim _{n \rightarrow+\infty}\left|D f^{n}(v)\right|=+\infty .
$$

Then for every Hölder continuous potential $\varphi: I \rightarrow \mathbb{R}$, the level-2 large deviation principle holds for $(f, \varphi)$ with respect to iterated preimages, periodic orbits, and Birkhoff averages, respectively, with the rate function $I^{\varphi}: \mathcal{M}(I) \rightarrow[0,+\infty]$ given by the following

$$
I^{\varphi}(\mu)= \begin{cases}P(f, \varphi)-\int_{I} \varphi d \mu-h_{\mu}(f) & \text { if } \mu \in \mathcal{M}(I, f) \\ +\infty & \text { if } \mu \in \mathcal{M}(I) \backslash \mathcal{M}(I, f) .\end{cases}
$$

Furthermore, for each convex open subset $\mathscr{G}$ of $\mathcal{M}(I)$ containing some invariant measure we have $\inf _{\mathscr{G}} I^{\varphi}=\inf _{\bar{G}} I^{\varphi}$, and

$$
\lim _{n \rightarrow+\infty} \frac{1}{n} \log \Theta_{n}(\mathscr{G})=\lim _{n \rightarrow+\infty} \frac{1}{n} \log \Theta_{n}\left(z_{0}\right)(\mathscr{G})=\lim _{n \rightarrow+\infty} \frac{1}{n} \log \Sigma_{n}(\mathscr{G})=-\inf _{\mathscr{G}} I^{\varphi} .
$$

Moreover, the above expression remains true replacing $\mathscr{G}$ by $\overline{\mathscr{G}}$.

To state our main result in the complex setting, for each complex rational map $f$ denote by $\operatorname{Crit}(f)$ the set of critical points of $f$, and by $J(f)$ the Julia set of $f$.

Theorem B. Let $f$ be one of the following:

1. An at most finitely renormalizable complex polynomial of degree at least 2 , without neutral cycles and such that for each critical value $v$ of $f$ we have

$$
\lim _{n \rightarrow+\infty}\left|D f^{n}(v)\right|=+\infty ;
$$

2. A complex rational map of degree at least 2, without parabolic cycles and such that for every critical value $v$ of $f$ we have

$$
\sum_{n=1}^{+\infty} \frac{1}{\left|D f^{n}(v)\right|}<+\infty
$$

Then for every Hölder continuous potential $\varphi: J(f) \rightarrow \mathbb{R}$, the level-2 large deviation principle holds for $(f, \varphi)$ with respect to iterated preimages, periodic orbits, and Birkhoff averages, respectively, with the rate function $I^{\varphi}: \mathcal{M}(J(f)) \rightarrow[0,+\infty]$ given by the following

$$
I^{\varphi}(\mu)= \begin{cases}P(f, \varphi)-\int_{J(f)} \varphi d \mu-h_{\mu}(f) & \text { if } \mu \in \mathcal{M}(J(f), f) ; \\ +\infty & \text { if } \mu \in \mathcal{M}(J(f)) \backslash \mathcal{M}(J(f), f) .\end{cases}
$$

Furthermore, for each convex open subset $\mathscr{G}$ of $\mathcal{M}(J(f))$ containing some invariant measure we have $\inf _{\mathscr{G}} I^{\varphi}=\inf _{\overline{G G}} I^{\varphi}$, and

$$
\lim _{n \rightarrow+\infty} \frac{1}{n} \log \Theta_{n}(\mathscr{G})=\lim _{n \rightarrow+\infty} \frac{1}{n} \log \Theta_{n}\left(z_{0}\right)(\mathscr{G})=\lim _{n \rightarrow+\infty} \frac{1}{n} \log \Sigma_{n}(\mathscr{G})=-\inf _{\mathscr{G}} I^{\varphi} .
$$

Moreover, the above expression remains true replacing $\mathscr{G}$ by $\bar{G}$.

Recall that for an integer $s \geq 2$, a complex polynomial $f$ is renormalizable of period $s$, if there are Jordan disks $U$ and $V$ in $\mathbb{C}$, such that $\bar{U} \subset V$ and such that the following hold:

- $f^{s}: U \rightarrow V$ is proper of degree at least 2 ; 
- The set $\left\{z \in U: f^{s n}(z) \in U\right.$ for all $\left.n=1,2, \ldots\right\}$ is a connected and it is strictly contained in $J(f)$;

- For each critical point $c$ of $f$ there exists at most one $j$ in $\{0,1, \ldots, s-1\}$ such that $c$ is $f^{j}(U)$.

We say that $f$ is infinitely renormalizable if there are infinitely many integers $s \geq 2$ such that $f$ is renormalizable of period $s$.

1.3. Organization of the proof. The paper is organized as follows. In 12 we state a version of Theorems $\mathrm{A}$ and $\mathrm{B}$ that holds for a more general class of maps; it is stated as the "Main Theorem". After deriving Theorems A and B from the Main Theorem and known results in $\$ 2.1$, we sate our main technical result as the "Key Lemma" in $\$ 2.2$ where we also derive the Main Theorem from it. In 93 , we give the proof of the Key Lemma.

1.4. Acknowledgments. The main idea of this paper came to the author after several discussions with Juan Rivera-Letelier on his a joint work with Henri Comman, see [CRL11. I am very grateful to him for those stimulating conversations and for his useful comments and corrections to an earlier version of this paper.

\section{A REDUCTION}

We start this section stating a version of Theorems $\mathrm{A}$ and $\mathrm{B}$ that holds for a more general class of maps; it is stated as the "Main Theorem". In $\$ 2.1$ we derive Theorems $\mathrm{A}$ and $\mathrm{B}$ as a direct consequence of the Main Theorem and known results. In $\$ 2.2$ we state our more general result as the "Key Lemma", and we derive the Main Theorem from it.

To state our main theorem, we introduce a class of interval maps that includes non-degenerate smooth maps as special cases. Let $I$ be a compact interval of $\mathbb{R}$. For a differentiable map $f: I \rightarrow I$, a point of $I$ is critical if the derivative of $f$ vanishes at it. Denote by $\operatorname{Crit}(f)$ the set of critical points of $f$. A differentiable interval map $f: I \rightarrow I$ is of class $C^{3}$ with non-flat critical points, if it has a finite number of critical points and if:

- The map $f$ is of class $C^{3}$ outside $\operatorname{Crit}(f)$;

- For each critical point $c$ of $f$ there exists a number $\ell_{c}>1$ and diffeomorphisms $\phi$ and $\psi$ of $\mathbb{R}$ of class $C^{3}$, such that $\phi(c)=\psi(f(c))=0$, and such that on a neighborhood of $c$ on $I$, we have

$$
|\psi \circ f|=|\phi|^{\ell_{c}} .
$$

Note that each smooth non-degenerate interval map is of class $C^{3}$ with non-flat critical points, and that each map of class $C^{3}$ with non-flat critical points is continuously differentiable.

For an interval map of class $C^{3}$ with non-flat critical points $f$, denote by $\operatorname{dom}(f)$ the interval on which $f$ is defined, and denote by dist the distance on $\operatorname{dom}(f)$ induced by the norm distance on $\mathbb{R}$. On the other hand, for a complex rational map $f$ we use $\operatorname{dom}(f)$ to denote the Riemann sphere $\overline{\mathbb{C}}$, which we endow with the spherical metric, that we also denote by dist. In both, the real and complex setting, for a subset $W$ of $\operatorname{dom}(f)$ we use $\operatorname{diam}(W)$ to denote the diameter of $W$ with respect to dist. 
Definition 2.1. Let $f$ be either a complex rational map, or an interval map of class $C^{3}$ with non-flat critical points. The Julia set $J(f)$ of $f$ is the complement of the largest open subset of $\operatorname{dom}(f)$ on which the family of iterates of $f$ is normal.

If $f$ is a complex rational map of degree at least 2 , then $J(f)$ is a perfect set that is equal to the closure of repelling periodic points. Moreover, $J(f)$ is completely invariant and $f$ is topologically exact on $J(f)$. We denote by $\mathscr{A}_{\mathbb{C}}$ the collection of all rational maps of degree at least 2 .

In contrast with the complex setting, the Julia set of an interval map of class $C^{3}$ with non-flat critical points might be empty, reduced to a single point, or might not be completely invariant. In that follows, we denote by $\mathscr{A} \mathbb{R}$ the collection of interval maps of class $C^{3}$ with non-flat critical points, whose Julia set contains at least 2 points and is completely invariant. Note that if $f: I \rightarrow I$ is a non-degenerate smooth map that is topologically exact, then $J(f)=I$ and $f$ is in $\mathscr{A} \mathbb{R}$. On the other hand, if $f$ is an interval map in $\mathscr{A}_{\mathbb{R}}$ that is topologically exact on $J(f)$, then $J(f)$ has no isolated points. For more background on the theory of Julia sets, see for example dMvS93, for the real setting, and [CG93, Mil06] for the complex setting.

Throughout the rest of this article we put $\mathscr{A}:=\mathscr{A}_{\mathbb{R}} \cup \mathscr{A}_{\mathbb{C}}$ and for each $f$ in $\mathscr{A}$ we restrict the action of $f$ to its Julia set. In particular, the topological pressure of $f$ is defined through measures supported on $J(f)$ and equilibrium states are supported on $J(f)$.

Definition 2.2. For $\beta>0$, a map $f$ in $\mathscr{A}$ satisfies the Polynomial Shrinking Condition with exponent $\beta$, if there exist constants $\rho_{0}>0$ and $C_{0}>0$ such that for every $x$ in $J(f)$, every integer $n \geq 1$, and every connected component $W$ of $f^{-n}\left(B\left(x, \rho_{0}\right)\right)$, we have

$$
\operatorname{diam}(W) \leq C_{0} n^{-\beta} .
$$

Main Theorem. Let $\beta>1$, and let $f$ be a map in $\mathscr{A}$ satisfying the Polynomial Shrinking Condition with exponent $\beta$. Suppose furthermore in the real case that $f$ is topologically exact on its Julia set. Then for each $\alpha$ in $\left(\beta^{-1}, 1\right]$, and every Hölder continuous potential $\varphi: J(f) \rightarrow \mathbb{R}$ of exponent $\alpha$, the level-2 large deviation principle holds for $(f, \varphi)$ with respect to iterated preimages, periodic orbits, and Birkhoff averages, respectively, with the rate function $I^{\varphi}: \mathcal{M}(J(f)) \rightarrow[0,+\infty]$ given by the following

$$
I^{\varphi}(\mu)= \begin{cases}P(f, \varphi)-\int_{J(f)} \varphi d \mu-h_{\mu}(f) & \text { if } \mu \in \mathcal{M}(J(f), f) \\ +\infty & \text { if } \mu \in \mathcal{M}(J(f)) \backslash \mathcal{M}(J(f), f) .\end{cases}
$$

Furthermore, for each convex open subset $\mathscr{G}$ of $\mathcal{M}(J(f))$ containing some invariant measure we have $\inf _{\mathscr{G}} I^{\varphi}=\inf _{\bar{G}} I^{\varphi}$, and

$$
\lim _{n \rightarrow+\infty} \frac{1}{n} \log \Theta_{n}(\mathscr{G})=\lim _{n \rightarrow+\infty} \frac{1}{n} \log \Theta_{n}\left(z_{0}\right)(\mathscr{G})=\lim _{n \rightarrow+\infty} \frac{1}{n} \log \Sigma_{n}(\mathscr{G})=-\inf _{\mathscr{G}} I^{\varphi} .
$$

Moreover, the above expression remains true replacing $\mathscr{G}$ by $\overline{\mathscr{G}}$.

Remark 2.3. In real case, our arguments and results can be generalized without change to the following. Let $f$ be an interval map in $\mathscr{A}_{\mathbb{R}}$ that is topologically exact on its Julia set, and let $\varphi: J(f) \rightarrow \mathbb{R}$ be a Hölder continuous function that is hyperbolic for $f$. If there is a dense vector subspace $\mathscr{W}$ of Hölder continuous functions defined on $J(f)$ such that for every $\psi \in \mathscr{W}$, the function $\varphi+\psi$ is hyperbolic for $f$, then the level-2 large deviation principle holds for $(f, \varphi)$ with respect to 
iterated preimages, periodic orbits, and Birkhoff averages, respectively, with the rate function $I^{\varphi}: \mathcal{M}(J(f)) \rightarrow[0,+\infty]$ given by the following

$$
I^{\varphi}(\mu)= \begin{cases}P(f, \varphi)-\int_{J(f)} \varphi d \mu-h_{\mu}(f) & \text { if } \mu \in \mathcal{M}(J(f), f) ; \\ +\infty & \text { if } \mu \in \mathcal{M}(J(f)) \backslash \mathcal{M}(J(f), f) .\end{cases}
$$

Furthermore, for each convex open subset $\mathscr{G}$ of $\mathcal{M}(J(f))$ containing some invariant measure we have $\inf _{\mathscr{G}} I^{\varphi}=\inf _{\bar{G}} I^{\varphi}$, and

$$
\lim _{n \rightarrow+\infty} \frac{1}{n} \log \Theta_{n}(\mathscr{G})=\lim _{n \rightarrow+\infty} \frac{1}{n} \log \Theta_{n}\left(z_{0}\right)(\mathscr{G})=\lim _{n \rightarrow+\infty} \frac{1}{n} \log \Sigma_{n}(\mathscr{G})=-\inf _{\mathscr{G}} I^{\varphi} .
$$

Moreover, the above expression remains true replacing $\mathscr{G}$ by $\overline{\mathscr{G}}$.

2.1. Proofs of Theorems $\mathbf{A}$ and $\mathbf{B}$ assuming the Main Theorem. First, we recall the definition of "backward contracting property" which was first introduced in [RL07] for the complex case, and in BRLSvS08] for the real case. For each map $f$ in $\mathscr{A}$, put

$$
\operatorname{CV}(f):=f(\operatorname{Crit}(f)) \text { and } \operatorname{Crit}^{\prime}(f):=\operatorname{Crit}(f) \cap J(f) .
$$

For a subset $V$ of $\operatorname{dom}(f)$, and an integer $m \geq 1$, each connected component of $f^{-m}(V)$ is a pull-back of by $f^{m}$. A pull-back $W$ of $V$ by $f^{n}$ is diffeomorphic if $f^{n}$ maps $W$ diffeomorphically onto a connected component of $V$, and it is nondiffeomorphic otherwise.

For every $c$ in $\operatorname{Crit}(f)$ and $\delta>0$, denote by $\widetilde{B}(c, \delta)$ the pull-back of $B(f(c), \delta)$ by $f$ that contains $c$.

Definition 2.4. Given a constant $r>1$, a map $f$ in $\mathscr{A}$ is backward contracting with constant $r$, if there exists $\delta_{0}>0$ such that for every $c$ in $\operatorname{Crit}^{\prime}(f)$, every $\delta$ in $\left(0, \delta_{0}\right)$, every integer $n \geq 0$, and every component $W$ of $f^{-n}(\widetilde{B}(c, r \delta))$, we have that

$$
\operatorname{dist}(W, \mathrm{CV}(f)) \leq \delta \text { implies } \operatorname{diam}(W)<\delta .
$$

If for each $r>1$ the map $f$ is backward contracting with constant $r$, then $f$ is backward contracting.

A map $f$ in $\mathscr{A}$ is expanding away from critical points, if for every neighborhood $V$ of $\mathrm{Crit}^{\prime}(f)$ the map $f$ is uniformly expanding on the set

$$
K(V):=\left\{z \in J(f): f^{i}(z) \notin V \text { for all } i \geq 0\right\} .
$$

In other words, there exist $C>0$ and $\lambda>1$ such that for every $z$ in $K(V)$ and $n \geq 0$, we have $\left|D f^{n}(z)\right| \geq C \lambda^{n}$.

Fact 2.5 ([RLS10], Theorem A). For each map $f$ in $\mathscr{A}$ and each $\beta>0$, there exists $r>1$ such that the following property holds. If $f$ is backward contracting with constant $r$ and is expanding away from critical points, then $f$ satisfies the Polynomial Shrinking Condition with exponent $\beta$.

Proof of Theorem A. By BRLSvS08, Theorem 1], the map $f$ is backward contracting and by Mañe's theorem $f$ is expanding away from critical points, see for example dMvS93. Then Fact 2.5 implies that for each $\beta>1$ the map $f$ satisfies the Polynomial Shrinking Condition with exponent $\beta$. So the desired assertions are direct consequences of the Main Theorem. 
Proof of Theorem B. By either [LS10, Theorem A] or [RL07, Theorem A], the map $f$ is backward contracting, and by either [KvS09] or [RL07, Corollary 8.3], it is expanding away from critical points. Then Fact 2.5 implies that for each $\beta>1$ the map $f$ satisfies the Polynomial Shrinking Condition with exponent $\beta$. So the desired assertions are direct consequences of the Main Theorem.

2.2. A reduction. In this subsection we prove the Main Theorem assuming the following key lemma, whose proof occupies the rest of this article.

Given an interval map $f$ in $\mathscr{A} \mathbb{R}$, a continuous function $\varphi: J(f) \rightarrow \mathbb{R}$ is said to be hyperbolic for $f$, if for some integer $n \geq 1$ the function $S_{n}(\varphi):=\sum_{i=0}^{n-1} \varphi \circ f^{i}$ satisfies $\sup _{J(f)} \frac{1}{n} S_{n}(\varphi)<P(f, \varphi)$.

Let $f$ be a map in $\mathscr{A}$. Given a measurable function $g: J(f) \rightarrow[0,+\infty)$, a probability measure $\mu$ supported on $J(f)$ is $g$-conformal for $f$, if for every measurable set $A \subset J(f)$ on which $f$ is injective, we have

$$
\mu(f(A))=\int_{A} g d \mu .
$$

Lemma 2.6. Let $f$ be a map in $\mathscr{A}$ that is topologically exact on its Julia set. Then for every Hölder continuous function $\varphi: J(f) \rightarrow \mathbb{R}$ that is hyperbolic for $f$, there exist an atom-free $\exp (P(f, \varphi)-\varphi)$-conformal measure $\mu$ for $f$, and a unique equilibrium state $\nu$ of $f$ for the potential $\varphi$. Moreover, $\nu$ is absolutely continuous with respect to $\mu$.

Proof. In the complex case, the assertions are a direct consequence of DU91, Theorem] and of [IRRL12, Proposition 3.1]. In the real setting, the assertions follow directly from [LRL12a, Theorem A] and Dob13, Theorem 6] or LRL12a, Theorem B].

Key Lemma. Let $f$ be an interval map in $\mathscr{A}_{\mathbb{R}}$ that is topologically exact on its Julia set $J(f)$. Then for every Hölder continuous function $\varphi: J(f) \rightarrow \mathbb{R}$ that is hyperbolic for $f$, the following properties hold:

1. For every point $x_{0}$ in $J(f)$, we have

$$
P(f, \varphi)=\lim _{n \rightarrow+\infty} \frac{1}{n} \log \sum_{y \in f^{-n}\left(x_{0}\right)} \exp \left(S_{n}(\varphi)(y)\right)
$$

2. If for every integer $n \geq 1$, put $\operatorname{Per}_{n}(f):=\left\{p \in J(f): f^{n}(p)=p\right\}$, then we have

$$
P(f, \varphi)=\lim _{n \rightarrow+\infty} \frac{1}{n} \log \sum_{p \in \operatorname{Per}_{n}(f)} \exp \left(S_{n}(\varphi)(p)\right) .
$$

3. Let $\nu$ be the unique equilibrium state of $f$ for the potential $\varphi$ given by Lemma 2.6. Then for every Hölder continuous function $\psi: J(f) \rightarrow \mathbb{R}$ such that $\varphi+\psi$ is hyperbolic for $f$, we have

$$
\lim _{n \rightarrow+\infty} \frac{1}{n} \log \int \exp \left(S_{n}(\psi)\right) d \nu=P(f, \varphi+\psi)-P(f, \varphi) .
$$

In order to prove our Main Theorem, we also need the following lemma, which is a variant of a general result of Kifer in Kif90.

Lemma 2.7 (Theorem C, CRL11]). Let $X$ be a compact metrizable topological space, and let $T: X \rightarrow X$ be a continuous map such that the measure-theoretic entropy of $T$, as a function defined on $\mathcal{M}(X, T)$ is finite and upper semi-continuous. 
Fix $\varphi \in C(X)$, and let $\mathcal{W}$ be a dense vector subspace of $C(X)$ such that for each $\phi \in \mathcal{W}$ there is a unique equilibrium state of $T$ for the potential $\varphi+\phi$. Let $I^{\varphi}$ : $\mathcal{M}(X) \rightarrow[0,+\infty]$ be the function defined by

$$
I^{\varphi}(\mu)= \begin{cases}P(T, \varphi)-\int_{X} \varphi d \mu-h_{\mu}(T) & \text { if } \mu \in \mathcal{M}(X, T) ; \\ +\infty & \text { if } \mu \in \mathcal{M}(X) \backslash \mathcal{M}(X, T) .\end{cases}
$$

Then every sequence $\left(\Omega_{n}\right)_{n \geq 1}$ of Borel probability measures on $\mathcal{M}(X)$ such that for every $\phi \in \mathcal{W}$

$$
\lim _{n \rightarrow+\infty} \frac{1}{n} \log \int_{\mathcal{M}(X)} \exp \left(n \int_{X} \phi d \mu\right) d \Omega_{n}(\mu)=P(T, \varphi+\phi)-P(T, \varphi),
$$

satisfies a large deviation principle with rate function $I^{\varphi}$, and it converges in the weak* topology to the Dirac mass supported on the unique equilibrium state of $T$ for the potential $\varphi$. Furthermore, for each convex and open subset $\mathscr{G}$ of $\mathcal{M}(X)$ containing some invariant measure, we have

$$
\lim _{n \rightarrow \infty} \frac{1}{n} \log \Omega_{n}(\mathscr{G})=\lim _{n \rightarrow \infty} \frac{1}{n} \log \Omega_{n}(\overline{\mathscr{G}})=-\inf _{\mathscr{G}} I^{\varphi}=-\inf _{\overline{\mathscr{G}}} I^{\varphi} .
$$

Proof of the Main Theorem assuming the Key Lemma. Let $\mathscr{P}$ be the collection of all Lipschitz continuous function defined on $J(f)$. Then $\mathscr{P}$ is a dense vector subspace of $C(J(f))$. By [LRL12b, Main Theorem] we have that $\varphi$ is hyperbolic for $f$, and that for every Lipschitz continuous function $\psi: J(f) \rightarrow \mathbb{R}$ we have $\varphi+\psi$ is also hyperbolic for $f$. By Lemma 2.6 there is a unique equilibrium state $\nu_{\varphi}$ of $f$ for the potential $\varphi$, and for every $\psi \in \mathscr{P}$ there is a unique equilibrium state of $f$ for the potential $\varphi+\psi$.

In the complex case, the proof of [CRL11, Theorem B] can be adapted to yield the Main Theorem, although there it is assumed that $f$ satisfies the "topological Collet-Eckmann" condition.

To prove assertions in the real setting, fix $\psi \in \mathscr{P}$. For the sequence $\left(\Theta_{n}\right)_{n \geq 1}$ associated to periodic points we have

$$
\begin{aligned}
& \int_{\mathcal{M}(J(f))} \exp \left(n \int_{J(f)} \psi d \mu\right) d \Theta_{n}(\mu) \\
&=\frac{\sum_{p \in \operatorname{Per}_{n}} \exp \left(S_{n}(\varphi)(p)\right) \exp \left(n \int_{J(f)} \psi d F_{n}(p)\right)}{\sum_{p^{\prime} \in \operatorname{Per}_{n}} \exp \left(S_{n}(\varphi)\left(p^{\prime}\right)\right)} \\
&=\frac{\sum_{p \in \operatorname{Per}_{n}} \exp \left(S_{n}(\varphi+\psi)(p)\right)}{\sum_{p^{\prime} \in \operatorname{Per}_{n}} \exp \left(S_{n}(\varphi)\left(p^{\prime}\right)\right)} .
\end{aligned}
$$

Analogously, for the sequence $\left(\Theta_{n}\left(x_{0}\right)\right)_{n \geq 1}$ associated to the iterated preimages of a point $x_{0} \in J(f)$, we have

$$
\int_{\mathcal{M}(J(f))} \exp \left(n \int_{J(f)} \psi d \mu\right) d \Theta_{n}\left(x_{0}\right)(\mu)=\frac{\sum_{x \in f^{-n}\left(x_{0}\right)} \exp \left(S_{n}(\varphi+\psi)(x)\right)}{\sum_{y \in f^{-n}\left(x_{0}\right)} \exp \left(S_{n}(\varphi)(y)\right)} ;
$$

for the the sequence $\left(\Sigma_{n}\right)_{n \geq 1}$ associated to the Birkhoff averages we have

$$
\int_{\mathcal{M}(J(f))} \exp \left(n \int_{J(f)} \psi d \mu\right) d \Sigma_{n}(\mu)=\int_{J(f)} \exp \left(S_{n}(\psi)\right) d \nu_{\varphi} .
$$


Therefore, the Key Lemma implies that (2.1) holds with $\phi=\psi$, and with $\left(\Omega_{n}\right)_{n \geq 1}$ replaced by each of the sequences $\left(\Theta_{n}\left(x_{0}\right)\right)_{n \geq 1},\left(\Theta_{n}\right)_{n \geq 1}$ and $\left(\Sigma_{n}\right)_{n \geq 1}$, respectively. On the other hand, the topological entropy of $f$ is finite, and the measure-theoretic entropy of $f$ is upper semi-continuous, see for example [BK98, Kel98. Consequently, the assertion of Main theorem follows from Lemma 2.7. The proof is complete.

\section{Proof of the Key Lemma}

Given an interval map $f$ in $\mathscr{A}_{\mathbb{R}}$, and a continuous function $\phi: J(f) \rightarrow \mathbb{R}$, denote by $\mathcal{L}_{\phi}$ the Ruelle-Perron-Frobenius operator, acting on the space of bounded functions defined on $J(f)$ and taking values in $\mathbb{C}$, as follows,

$$
\mathcal{L}_{\phi}(h)(x):=\sum_{y \in f^{-1}(x)} \exp (\phi(y)) h(y) .
$$

Moreover, put $\widehat{\mathcal{L}}_{\phi}:=\exp (-P(f, \phi)) \mathcal{L}_{\phi}$.

The following proposition is the main ingredient to prove parts 1 and 3 of the Key Lemma.

Proposition 3.1 (Proposition 2.1, [LRL12a]). Let $f$ be an interval map in $\mathscr{A} \mathbb{R}$ that is topologically exact on its Julia set $J(f)$, and denoted by $\mathbf{1}$ the function defined on $J(f)$ that is constant equal to 1 . Then for every Hölder continuous function $\phi: J(f) \rightarrow \mathbb{R}$ that is hyperbolic for $f$, and every $\varepsilon>0$, we have for every sufficiently large integer $n$

$$
\exp (-\varepsilon n) \leq \inf _{J(f)} \widehat{\mathcal{L}}_{\phi}^{n}(\mathbf{1}) \leq \sup _{J(f)} \widehat{\mathcal{L}}_{\phi}^{n}(\mathbf{1}) \leq \exp (\varepsilon n) .
$$

We also need the following lemma.

Lemma 3.2 (Lemma 5.1, LRL12a]). Let $f$ be an interval map in $\mathscr{A} \mathbb{R}$, and let $\phi$ : $J(f) \rightarrow \mathbb{R}$ be Hölder continuous that is hyperbolic for $f$. If $\mu$ is a $\exp (P(f, \phi)-\phi)$ conformal measure for $f$, then for every function $h \in L^{1}(\mu)$, we have

$$
\int_{J(f)} \widehat{\mathcal{L}}_{\phi}(h) d \mu=\int_{J(f)} h d \mu .
$$

Proof of parts 1 and 3 of the Key Lemma. Part 1 is a direct consequence of Proposition 3.1 with $\phi=\varphi$. To prove part 3, note that by Lemma 2.6 there is a $\exp (P(f, \varphi)-\varphi)$-conformal measure $\mu$ for $f$. Moreover, $\nu$ is absolutely continuous with respect to $\mu$. Let $h_{\nu}: J(f) \rightarrow \mathbb{R}$ be a density function of $\nu$ with respect to $\mu$, so that $\nu=h_{\nu} \mu$. By Lemma 3.2, we have

$$
\begin{aligned}
\int_{J(f)} \exp \left(S_{n}(\psi)\right) d \nu & =\int_{J(f)} \exp \left(S_{n}(\psi)\right) h_{\nu} d \mu=\int_{J(f)} \widehat{\mathcal{L}}_{\varphi}^{n}\left(\exp \left(S_{n}(\psi)\right) h_{\nu}\right) d \mu \\
& =\exp \left(-n P(f, \varphi) \int_{J(f)} \mathcal{L}_{\varphi}^{n}\left(\exp \left(S_{n}(\psi)\right) h_{\nu}\right) d \mu\right. \\
& =\exp \left(-n P(f, \varphi) \int_{J(f)} \mathcal{L}_{\varphi+\psi}^{n}\left(h_{\nu}\right) d \mu\right. \\
& =\exp (n(P(f, \varphi+\psi)-P(f, \varphi))) \int_{J(f)} \widehat{\mathcal{L}}_{\varphi+\psi}\left(h_{\nu}\right) d \mu
\end{aligned}
$$


By Proposition 3.1 with $\phi=\varphi+\psi$, for every $\varepsilon>0$ we have for every integer $n \geq 1$ large enough

$$
\exp (-\varepsilon n) h_{\nu} \leq \inf _{J(f)} \widehat{\mathcal{L}}_{\varphi+\psi}^{n}\left(h_{\nu}\right) \leq \sup _{J(f)} \widehat{\mathcal{L}}_{\varphi+\psi}\left(h_{\nu}\right) \leq \exp (\varepsilon n) h_{\nu}
$$

It follows that

$$
\begin{aligned}
\exp (-\varepsilon n)=\int_{J(f)} \exp (-\varepsilon n) h_{\nu} d \mu \leq \int_{J(f)} \widehat{\mathcal{L}}_{\varphi+\psi}\left(h_{\nu}\right) d \mu \\
\quad \leq \int_{J(f)} \exp (-\varepsilon n) h_{\nu} d \mu=\exp (\varepsilon n) .
\end{aligned}
$$

Therefore, for every sufficiently large integer $n$ we have

$P(f, \varphi+\psi)-P(f, \varphi)-\varepsilon \leq \frac{1}{n} \log \int_{J(f)} \exp \left(S_{n}(\psi)\right) d \mu_{\varphi} \leq P(f, \varphi+\psi)-P(f, \varphi)+\varepsilon$.

Letting $\varepsilon \rightarrow 0$ we complete the proof of part 3 of the Key Lemma.

The rest of this section is devoted to prove part 2 of the Key Lemma. The proof, which is given at the end of this section, depends on several lemmas.

Lemma 3.3. Let $f$ be an interval map in $\mathscr{A}_{\mathbb{R}}$ that is topologically exact on $J(f)$. Then, for every $\kappa>0$ there is $\delta_{1}>0$ such that the following holds. For every interval $J \subset I$ such that $\partial J \subset J(f)$ and $|J| \leq \delta_{1}$, every integer $n \geq 0$, and every pull-back $W$ of $J$ by $f^{n}$, we have $|W| \leq \kappa$.

Proof. See the proof of RL12, Lemma A.2] for example. There it is assumed that all cycles are hyperbolic repelling, but the proof can be adapted to yield the lemma.

Lemma 3.4 (Lemma 2.3, LRL12a]). Let $f$ be an interval map in $\mathscr{A}_{\mathbb{R}}$ and let $\varphi: J(f) \rightarrow \mathbb{R}$ be a Hölder continuous function. Then for every integer $N \geq 1$ and there is a constant $C>1$ such that the function $\widetilde{\varphi}:=\frac{1}{N} S_{N}(\varphi)$ satisfies the following properties:

1. The function $\widetilde{\varphi}$ is Hölder continuous of the same exponent as $\varphi, P(f, \widetilde{\varphi})=$ $P(f, \varphi)$, and $\varphi$ and $\widetilde{\varphi}$ share the same equilibrium states;

2. For every integer $n \geq 1$, we have

$$
\sup _{J(f)}\left|S_{n}(\widetilde{\varphi})-S_{n}(\varphi)\right| \leq C .
$$

Lemma 3.5 (Lemma 3.2, [LRL12b]). For each interval map $f: I \rightarrow I$ in $\mathscr{A}_{\mathbb{R}}$ there is $\varepsilon>0$ such that the following property holds. Let $J_{0}$ be an interval contained in $I$ satisfying $\left|J_{0}\right| \leq \varepsilon$, let $n \geq 1$ be an integer, and let $J$ be a pull-back of $J_{0}$ by $f^{n}$, such that for each $j$ in $\{1, \ldots, n\}$ the pull-back of $J_{0}$ by $f^{j}$ containing $f^{n-j}(J)$ has length bounded from above by $\varepsilon$. If in addition the closure of $J$ is contained in the interior of $I$, then $f^{n}(\partial J) \subset \partial J_{0}$.

Given an integer $n \geq 1$ and a point $x$ in $\operatorname{dom}(f)$, a preimage $y$ of $x$ by $f^{n}$ is critical if $D f^{n}(y)=0$, and it is non-critical otherwise. The following lemma was proved in LRL12a, whose proof is based on Przytycki and Urbański's adaptation to one-dimensional maps of Katok-Pesin theory, see for example [PU10, §11.6]. 
Lemma 3.6 (Lemma 2.4, LRL12a]). Let $f$ be an interval map in $\mathscr{A}_{\mathbb{R}}$ that is topologically exact on $J(f)$, and let $\phi: J(f) \rightarrow \mathbb{R}$ be a Hölder continuous potential satisfying $\sup _{J(f)} \phi<P(f, \phi)$. Then for every point $x_{0}$ of $J(f)$ having infinitely many non-critical preimages, there is $\delta>0$ such that the following property holds: If for each integer $n \geq 1$ we denote by $\mathfrak{D}_{n}$ the collection of diffeomorphic pull-backs of $B\left(x_{0}, \delta\right)$ by $f^{n}$, then

$$
\liminf _{n \rightarrow+\infty} \frac{1}{n} \log \sum_{W \in \mathfrak{D}_{n}} \inf _{W \cap J(f)} \exp \left(S_{n}(\phi)\right) \geq P(f, \phi) .
$$

In particular,

$$
\liminf _{n \rightarrow+\infty} \frac{1}{n} \log \sum_{x \in f^{-n}\left(x_{0}\right)} \exp \left(S_{n}(\phi)(x)\right) \geq P(f, \phi) .
$$

Throughout the rest of this section, fix $f$ and $\varphi$ as in the Key Lemma. Recall that for every integer $n \geq 1, \operatorname{Per}_{n}(f)=\left\{p \in J(f): f^{n}(p)=p\right\}$. Since $\varphi$ is hyperbolic for $f$, there is an integer $N \geq 1$ such that the function $\widetilde{\varphi}:=\frac{1}{N} S_{N}(\varphi)$ satisfies $\sup _{J(f)} \widetilde{\varphi}<P(f, \varphi)$. By part 1 of Lemma 3.4, the function $\widetilde{\varphi}$ is Hölder continuous and

$$
P(f, \widetilde{\varphi})=P(f, \varphi)>\sup _{J(f)} \widetilde{\varphi} .
$$

Lemma 3.7. $\liminf _{n \rightarrow+\infty} \frac{1}{n} \log \sum_{p \in \operatorname{Per}_{n}(f)} \exp \left(S_{n}(\widetilde{\varphi})(p)\right) \geq P(f, \widetilde{\varphi})$.

Proof. Let $x_{0}$ be a point of $J(f)$ that is not a boundary point of a connected component of $\mathbb{R} \backslash J(f)$. Assume in addition that for every $c$ in Crit $(f)$ and every integer $n \geq 1$ we have $f^{n}(c) \neq x_{0}$. Let $\delta>0$ be the constant given by Lemma 3.6 with $\phi=\widetilde{\varphi}$, and let $V \subset B\left(x_{0}, \delta\right)$ be a closed interval such that $\partial V \subset J(f)$ and such that $x_{0}$ is an interior point of $V$. Since $f$ is topologically exact on its Julia set, there is $s \geq 1$ such that $f^{s}(V) \supset J(f)$.

For each integer $n \geq 1$, denote by $\mathfrak{D}_{n}$ the collection of diffeomorphic pull-backs of $B\left(x_{0}, \delta\right)$ by $f^{n}$. For every integer $n>s$ and each $D \in \mathfrak{D}_{n-s}$, let $D^{\prime}$ be the connected component of $f^{-(n-s)}(V)$ contained in $D$. Note that $\partial D^{\prime} \subset J(f)$ and $f^{n-s}\left(D^{\prime}\right)=V$. Therefore $D^{\prime} \subset f^{n}\left(D^{\prime}\right)$, and so there is a point $p \in \operatorname{Per}_{n}(f)$ in $D^{\prime}$. It follows that for every $n>s$

$$
\begin{array}{r}
\frac{1}{n} \log \sum_{p \in \operatorname{Per}_{n}(f)} \exp \left(S_{n}(\widetilde{\varphi})(p)\right) \geq \frac{1}{n} \log \left(\sum_{D \in \mathfrak{D}_{n-s}} \sum_{p \in D^{\prime} \cap \operatorname{Per}_{n}(f)} \exp \left(S_{n}(\widetilde{\varphi})(p)\right)\right) \\
=\frac{1}{n} \log \left(\sum_{D \in \mathfrak{D}_{n-s}} \sum_{p \in D^{\prime} \cap \operatorname{Per}_{n}(f)}\left(\exp \left(S_{n-s}(\widetilde{\varphi})(p)+S_{s}(\widetilde{\varphi})\left(f^{n-s}(p)\right)\right)\right)\right) \\
\geq \frac{1}{n} \log \sum_{D \in \mathfrak{D}_{n-s}} \inf _{D^{\prime} \cap J(f)} \exp \left(S_{n-s}(\widetilde{\varphi})\right)+\frac{s}{n} \inf _{J(f)} \widetilde{\varphi} .
\end{array}
$$

By Lemma 3.6 we have

$$
\liminf _{n \rightarrow+\infty} \frac{1}{n} \log \sum_{D \in \mathfrak{D}_{n}} \inf _{D^{\prime} \cap J(f)} \exp \left(S_{n}(\widetilde{\varphi})\right) \geq P(f, \widetilde{\varphi}) .
$$


Hence,

$$
\liminf _{n \rightarrow+\infty} \frac{1}{n} \log \sum_{p \in \operatorname{Per}_{n}(f)} \exp \left(S_{n}(\widetilde{\varphi})(p)\right) \geq P(f, \widetilde{\varphi}),
$$

and we complete the proof.

Lemma 3.8. Let $\kappa>0$, and let $L \geq 1$ be an integer such that for every critical point $c$ and every $i$ in $\{1,2, \cdots, L\}$, we have either

$$
f^{i}(c) \notin B(\text { Crit, } \kappa) \text { or } f^{i}(c) \in \operatorname{Crit}(f) .
$$

Then for every interval $W$ and each integer $n \geq 1$ such that for every $i$ in $\{0,1, \cdots, n\}$ we have $\left|f^{i}(W)\right|<\kappa$, there are at most $2^{1+n / L}$ critical points of $f^{n}$ in $W$. In particular, there are at most $2^{2+n / L}$ maximal monotonic intervals of $f^{n}$ in $W$.

Proof. Fix an interval $W$ and an integer $n \geq 1$, such that for every $i$ in $\{0,1, \cdots, n\}$ we have $\left|f^{i}(W)\right|<\kappa$. Define an integer $s \geq 1$ and a strictly increasing sequence of integers $\left(n_{0}, \cdots, n_{s}\right)$ with $n_{0}=0$ and $n_{s}=n$, by induction as follows. Suppose $j \geq 0$ is an integer such that $n_{j} \leq n-1$ is already defined. If $n_{j}+L \geq n$ or if $n_{j}+L \leq n-1$ and for each $i$ in $\left\{n_{j}+L, \cdots, n-1\right\}$ the set $f^{i}(W)$ does not intersect $\operatorname{Crit}(f)$, then put $n_{j+1}:=n, s:=j+1$ and stop. Otherwise, let $n_{j+1}$ be the least integer $i$ in $\left\{n_{j}+L, \cdots, n-1\right\}$ such that $f^{i}(W) \cap \operatorname{Crit}(f) \neq \emptyset$.

Using the definitions of $L$ and our construction of $\left(n_{0}, \cdots, n_{s}\right)$, we know that and for every $i \in\{1, \cdots, s\}$ we have $n_{i}-n_{i-1} \geq L$ and the map $f^{n_{i}-n_{i-1}}$ has at most one critical point in $f^{n_{i-1}}(W)$. It follows that the map $f^{n}$ has at most $2^{s}$ critical points in $W$. Note that $s \leq 1+n / L$, we conclude that the number of maximal monotonic intervals of $f^{n}$ contained in $W$ less than $1+2^{s} \leq 2^{2+n / L}$.

Lemma 3.9. For every $\eta>1$ there is $\delta>0$ such that the following holds. Let $V$ be an interval intersecting $J(f)$ and such that $|V|<\delta$. For every integer $n \geq 1$ and each pull-back $W$ of $V$ by $f^{n}$ there are at most $\eta^{n}$ periodic points of $f$ of periodic $n$ in $W$.

Proof. Fix $\eta>1$ and let $L>1$ be large enough such that for every $n \geq L$ we have $2^{2+n / L}<\eta^{n}$. Let $\kappa>0$ be sufficiently small so that for every critical point $c$ and every $i$ in $\{1,2, \cdots, L\}$, we have either

$$
f^{i}(c) \notin B(\operatorname{Crit}(f), \kappa) \text { or } f^{i}(c) \in \operatorname{Crit}(f) .
$$

Let $\delta>0$ be the constant given by Lemma 3.3. Let $V$ be an interval intersecting $J(f)$ and such that $|V|<\delta$. By Lemma 3.8, for every $n \geq L$ and each pull-back $W$ of $V$ by $f^{n}$ there are at most $2^{2+n / L}$ maximal monotonic intervals of $f^{n}$ in $W$.

To complete the proof, it suffices to prove for every $n \geq 1$ and every interval $U$ such that $f^{n}$ is strictly monotonic on $U$, there is at most one point in $U \cap \operatorname{Per}_{n}(f)$. Otherwise, if there are two distinct points $p$ and $p^{\prime}$ in $U \cap \operatorname{Per}_{n}(f)$, then by assumption $f^{n}\left(\left[p, p^{\prime}\right]\right)=\left[p, p^{\prime}\right]$. It follows that for any integer $k \geq 1$ we have $f^{n k}\left(\left[p, p^{\prime}\right]\right)=\left[p, p^{\prime}\right] \not \supset J(f)$. This is a contradiction with our assumption that $f$ is topologically exact on its Julia set. The lemma is proved.

Proof of part 2 of the Key Lemma. In view of part 2 of Lemma 3.4 and of Lemma3.7. it suffices to show

$$
\limsup _{n \rightarrow+\infty} \frac{1}{n} \log \sum_{p \in \operatorname{Per}_{n}(f)} \exp \left(S_{n}(\widetilde{\varphi})(p)\right) \leq P(f, \widetilde{\varphi}) .
$$


Denote by $\varepsilon_{0}>0$ the constant given by Lemma 3.5. Fix $\varepsilon \in\left(0, \varepsilon_{0}\right)$ and let $\delta_{1}$ be the constant given by Lemma 3.3 with $\kappa=\varepsilon$. Let $\eta>1$ be given, and let $\delta>0$ be the constant given by Lemma 3.9. Put $r_{0}:=\min \left\{\delta_{1}, \delta\right\}$, and let $\mathcal{V}$ be a finite covering of $J(f)$ by intervals such that for every $V$ in $\mathcal{V}$ we have $|V|<r_{0}$ and $\partial V \subset J(f)$. Moreover, put $\partial \mathcal{V}:=\bigcup_{V \in \mathcal{V}} \partial V$. In view of [LRL12b, Lemma 2.8], see also [Prz90, Lemma 4], there is $C_{@}>0$ such that for every integer $n \geq 1$ we have

$$
\sum_{y \in f^{-n}(\partial \mathcal{V})} \exp \left(S_{n}(\widetilde{\varphi})(y)\right) \leq C_{@} \exp (n(P(f, \widetilde{\varphi})+\varepsilon)) .
$$

Fix an integer $n \geq 1$, and let $\mathcal{W}_{n}$ be collection of all pull-backs of elements of $\mathcal{V}$ by $f^{n}$. For every $W \in \mathcal{W}_{n}$, by Lemma 3.9 there are at most $\eta^{n}$ periodic points of $f$ of periodic $n$ in $W$, and by Lemma 3.3 for every $i$ in $\{0,1, \cdots, n-1\}$ we have $\left|f^{i}(W)\right|<\varepsilon$. Therefore, by Lemma 3.5 we have $f^{n}(\partial W) \subset \partial V$ if $W \cap \partial I=\emptyset$. On the other hand, there exist $C>1$ and $\alpha>0$ only dependent of $\widetilde{\varphi}$ such that for every $x, y \in W$ we have $\left|S_{n}(\widetilde{\varphi})(x)-S_{n}(\widetilde{\varphi})(y)\right| \leq C n \varepsilon^{\alpha}$. It follows that

$$
\begin{aligned}
& \sum_{p \in \operatorname{Per}_{n}(f)} \exp \left(S_{n}(\widetilde{\varphi})(p)\right)
\end{aligned}
$$

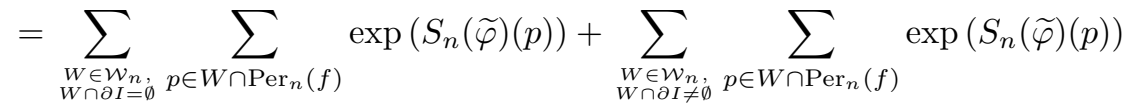

$$
\begin{aligned}
& \leq 2 \# \mathcal{V} \eta^{n} \exp \left(n \sup _{J(f)} \widetilde{\varphi}\right)+2 \eta^{n} \sum_{y \in f^{-n}(\partial \mathcal{V})} \exp \left(S_{n}(\widetilde{\varphi})(y)+C n \varepsilon^{\alpha}\right)
\end{aligned}
$$

Together with $\sup _{J(f)} \widetilde{\varphi}<P(f, \widetilde{\varphi})$ and (3.1), this implies

$$
\begin{aligned}
& \sum_{p \in \operatorname{Per}_{n}(f)} \exp \left(S_{n}(\widetilde{\varphi})(p)\right) \\
& <2 \# \mathcal{V} \eta^{n} \exp (n P(f, \widetilde{\varphi}))+2 C_{@} \eta^{n} \exp \left(C n \varepsilon^{\alpha}\right) \exp (n(P(f, \widetilde{\varphi})+\varepsilon)) \\
& \leq 2\left(\# \mathcal{V}+C_{@}\right) \eta^{n} \exp \left(n\left(P(f, \widetilde{\varphi})+C \varepsilon^{\alpha}+\varepsilon\right)\right) .
\end{aligned}
$$

Hence,

$$
\limsup _{n \rightarrow+\infty} \frac{1}{n} \log \sum_{p \in \operatorname{Per}_{n}(f)} \exp \left(S_{n}(\widetilde{\varphi})(p)\right) \leq \log \eta+P(f, \widetilde{\varphi})+C \varepsilon^{\alpha}+\varepsilon .
$$

Letting $\eta \rightarrow 1$ and $\varepsilon \rightarrow 0$, we have

$$
\limsup _{n \rightarrow+\infty} \frac{1}{n} \log \sum_{p \in \operatorname{Per}_{n}(f)} \exp \left(S_{n}(\widetilde{\varphi})(p)\right) \leq P(f, \widetilde{\varphi}),
$$

and the proof is complete.

\section{REFERENCES}

[AP06] V. Araújo and M. J. Pacifico. Large deviations for non-uniformly expanding maps. J. Stat. Phys., 125(2):415-457, 2006.

[BK98] Henk Bruin and Gerhard Keller. Equilibrium states for S-unimodal maps. Ergodic Theory Dynam. Systems, 18(4):765-789, 1998.

[BRLSvS08] H. Bruin, J. Rivera-Letelier, W. Shen, and S. van Strien. Large derivatives, backward contraction and invariant densities for interval maps. Invent. Math., 172(3):509-533, 2008 . 
[CG93] Lennart Carleson and Theodore W. Gamelin. Complex dynamics. Universitext: Tracts in Mathematics. Springer-Verlag, New York, 1993.

[Com09] Henri Comman. Strengthened large deviations for rational maps and full shifts, with unified proof. Nonlinearity, 22(6):1413-1429, 2009.

[CRL11] Henri Comman and Juan Rivera-Letelier. Large deviation principles for nonuniformly hyperbolic rational maps. Ergodic Theory Dynam. Systems, 31(2):321349, 2011.

[CT12] Yong Moo Chung and Hiroki Takahasi. Large deviation principle for BenedicksCarleson quadratic maps. Comm. Math. Phys., 315(3):803-826, 2012.

[CTY13] Vaughn Climenhaga, Daniel J. Thompson, and Kenichiro Yamamoto. Large deviations for systems with non-uniform structure. arXiv:1304.5497v1, 2013.

[Den96] Manfred Denker. Probability theory for rational maps. In Probability theory and mathematical statistics (St. Petersburg, 1993), pages 29-40. Gordon and Breach, Amsterdam, 1996.

[dMvS93] Welington de Melo and Sebastian van Strien. One-dimensional dynamics, volume 25 of Ergebnisse der Mathematik und ihrer Grenzgebiete (3) [Results in Mathematics and Related Areas (3)]. Springer-Verlag, Berlin, 1993.

[Dob13] Neil Dobbs. Pesin theory and equilibrium measures on the interval. arXiv:1304.3305v1, 2013.

[DU91] M. Denker and M. Urbański. Ergodic theory of equilibrium states for rational maps. Nonlinearity, 4(1):103-134, 1991.

[IRRL12] Irene Inoquio-Renteria and Juan Rivera-Letelier. A characterization of hyperbolic potentials of rational maps. Bull. Braz. Math. Soc. (N.S.), 43(1):99-127, 2012.

[Kel98] Gerhard Keller. Equilibrium states in ergodic theory, volume 42 of London Mathematical Society Student Texts. Cambridge University Press, Cambridge, 1998.

[Kif90] Yuri Kifer. Large deviations in dynamical systems and stochastic processes. Trans. Amer. Math. Soc., 321(2):505-524, 1990.

[KN92] Gerhard Keller and Tomasz Nowicki. Spectral theory, zeta functions and the distribution of periodic points for Collet-Eckmann maps. Comm. Math. Phys., 149(1):31-69, 1992.

[KvS09] Oleg Kozlovski and Sebastian van Strien. Local connectivity and quasi-conformal rigidity of non-renormalizable polynomials. Proc. Lond. Math. Soc. (3), 99(2):275296, 2009.

[LRL12a] Huaibin Li and Juan Rivera-Letelier. Equilibrium states of interval maps for hyperbolic potentials. arXiv:1210.6952 2 [math.DS], 2013.

[LRL12b] Huaibin Li and Juan Rivera-Letelier. Equilibrium states of weakly hyperbolic onedimensional maps for hölder potentials. arXiv:1210.0521 v2 [math.DS], 2012.

[LS10] HuaiBin Li and WeiXiao Shen. On non-uniform hyperbolicity assumptions in onedimensional dynamics. Sci. China Math., 53(7):1663-1677, 2010.

[Mel09] Ian Melbourne. Large and moderate deviations for slowly mixing dynamical systems. Proc. Amer. Math. Soc., 137(5):1735-1741, 2009.

[Mil06] John Milnor. Dynamics in one complex variable, volume 160 of Annals of Mathematics Studies. Princeton University Press, Princeton, NJ, third edition, 2006.

[MN08] Ian Melbourne and Matthew Nicol. Large deviations for nonuniformly hyperbolic systems. Trans. Amer. Math. Soc., 360(12):6661-6676, 2008.

[Prz90] Feliks Przytycki. On the Perron-Frobenius-Ruelle operator for rational maps on the Riemann sphere and for Hölder continuous functions. Bol. Soc. Brasil. Mat. (N.S.), 20(2):95-125, 1990.

[PS96] Mark Pollicott and Richard Sharp. Large deviations and the distribution of preimages of rational maps. Comm. Math. Phys., 181(3):733-739, 1996.

[PS09a] Mark Pollicott and Richard Sharp. Large deviations, fluctuations and shrinking intervals. Comm. Math. Phys., 290(1):321-334, 2009.

[PS09b] Mark Pollicott and Richard Sharp. Large deviations for intermittent maps. Nonlinearity, 22(9):2079-2092, 2009.

[PSY98] Mark Pollicott, Richard Sharp, and Michiko Yuri. Large deviations for maps with indifferent fixed points. Nonlinearity, 11(4):1173-1184, 1998. 
[PU10] Feliks Przytycki and Mariusz Urbański. Conformal fractals: ergodic theory methods, volume 371 of London Mathematical Society Lecture Note Series. Cambridge University Press, Cambridge, 2010.

[RBY08] Luc Rey-Bellet and Lai-Sang Young. Large deviations in non-uniformly hyperbolic dynamical systems. Ergodic Theory Dynam. Systems, 28(2):587-612, 2008.

[RL07] Juan Rivera-Letelier. A connecting lemma for rational maps satisfying a no-growth condition. Ergodic Theory Dynam. Systems, 27(2):595-636, 2007.

[RL12] Juan Rivera-Letelier. On the asymptotic expansion of maps with disconnected Julia set. 2012. arXiv: $1206.2376 \mathrm{v} 1$.

[RLS10] Juan Rivera-Letelier and Weixiao Shen. Statistical properties of one-dimensional maps under weak hyperbolicity assumptions. 2010. arXiv:1004.0230 v1.

[XF07] Hongqiang Xia and Xinchu Fu. Remarks on large deviation for rational maps on the Riemann sphere. Stoch. Dyn., 7(3):357-363, 2007.

[You90] Lai-Sang Young. Large deviations in dynamical systems. Trans. Amer. Math. Soc., 318(2):525-543, 1990.

Huaibin Li, Facultad de Matemáticas, Pontificia Universidad Católica de Chile, Avenida Vicuña Mackenna 4860, Santiago, Chile

E-mail address: matlihb@gmail.com 